Ann. Biol. anim. Bioch. Biophys., I965, 5 (3), 407-4Ir.

NOTE TECHNIQUE

\title{
MÉTHODE DE PRÉLÈVEMENT AUTOMATIQUE DE LA PHASE LIQUIDE DES CONTENUS DE RUMEN
}

\author{
B. GAUSSERES \\ Laboratoire des Métabolismes, \\ Centre national de Recherches zootechniques, Jouy-en-Josas (Seine-et-Oise)
}

L'échantillonnage des contenus de rumen in vivo est un problème complexe qui jusqu'ici n'a pas trouvé de solution satisfaisante. Dans les études relatives à certains produits terminaux de la digestion (acides aminés libres, ammoniac, acides gras volatils), on peut ne prélever que la phase liquide. La physiologie et le comportement alimentaire des ruminants sont tels que les études cinétiques exigent des prélèvements nombreux et rapprochés. En outre, l'ouverture des canules et les manipulations changent les conditions normales de la digestion (anaérobiose) ce qui risque de conduire à des résultats peu physiologiques.

Enfin, bien que non compartimenté, le rumen comporte des zones bien différenciées de digestion que l'on mélange lors de la constitution d'échantillons moyens représentatifs de l'ensemble du contenu. Il nous semble préférable (hormis certaines études particulières de bilan) de suivre les évolutions fermentaires en un point donné du rumen. Nous avons ainsi été amenés à mettre au point le dispositif suivant de prélèvement.

\section{Description de l'appareillage}

Le dispositif utilisé jusqu'ici pour des moutons se compose :

- d'un chronorelais permettant de fermer un circuit électrique pendant une durée réglable, le temps d'ouverture étant variable;

- d'une pompe à action péristaltique (Sigmamotor avec tête M'T 8);

- d'un collecteur de fractions (Seive) solidaire d'un chronodéclencheur et placé dans une enceinte refroidie à $0^{\circ} \mathrm{C}$;

- dsune crépine immergée dans le rumen. Cette crépine est maintenue dans une position déterminée.

Nous avons adopté jusqu'ici des cycles de fonctionnement de I heure compre- 
nant 16 minutes de fermeture du circuit électrique et 44 minutes d'ouverture. Quand le circuit est fermé, la pompe et le chronodéclencheur du collecteur fonctionnent. Ce dernier, fait déplacer le plateau du collecteur de façon telle qu'un tube est présenté toutes les 5 minutes, soit trois fois pendant les 16 minutes de fermeture. De cette

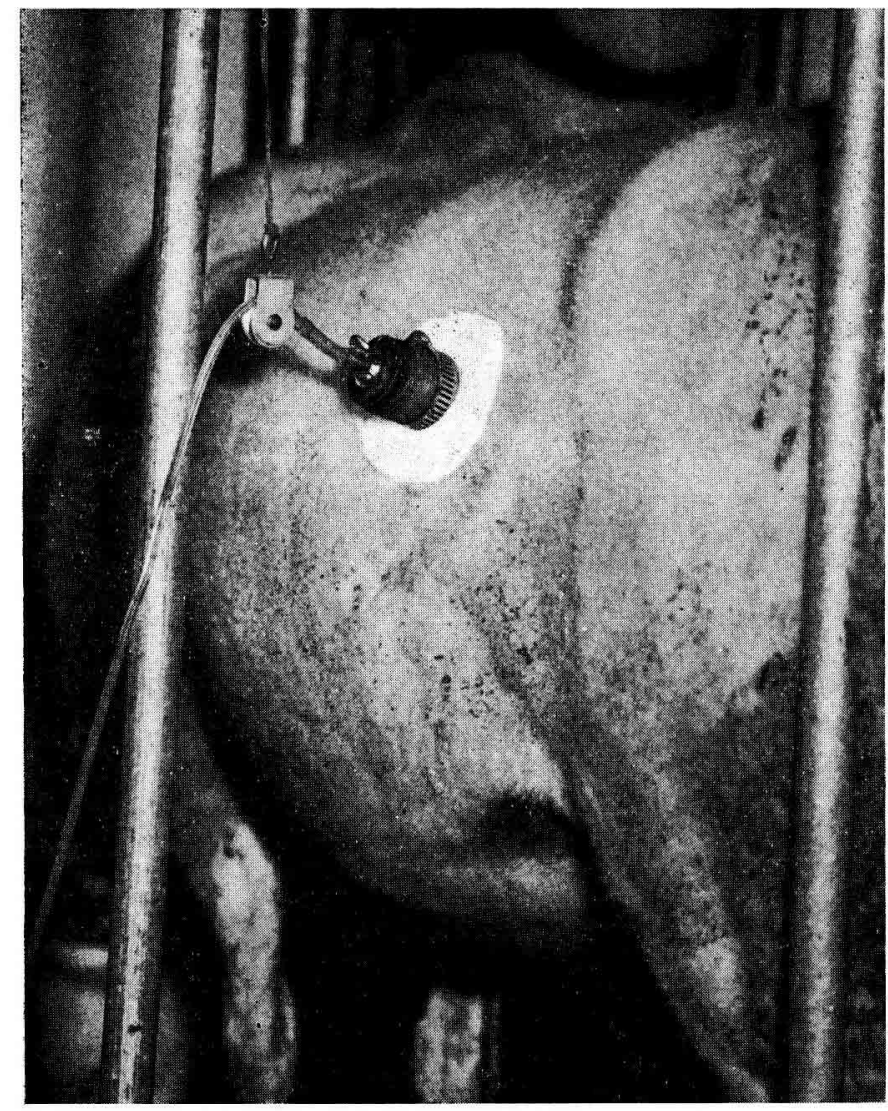

Hin. I. Racorlement du labe de prélivement de la pompe ì lanimal

façon, le premier tube d'un cycle $n$ est le même que le dernier tube du cycle $n$-I. Ce premier tube reçoit le jus correspondant au rinçage du circuit. Les deux tubes suivants contiennent le prélèvement proprement dit. La présentation du dernier tube se fait une minute avant l'ouverture du circuit (ce tube sera le premier du cycle suivant). I a pompe est réglée de façon à obtenir un débit de $\mathrm{I} \mathrm{ml} / \mathrm{mn}$ environ. Il est difficile d'obtenir un débit très constant pendant une période longue. Le volume mort du dispositif de prélèvement est d'environ $4 \mathrm{ml}$.

La crépine, fixée sur la canule, se compose d'un tube d'acier inoxydable coudé de I/4 de pouce de diamètre. La longueur du tube est fonction de la taille de l'animal. Elle doit être telle que le dispositif une fois fixé sur la canule, il existe un espace d'environ $3 \mathrm{~cm}$ entre son extrémité et la paroi du rumen. Le tube est relié à chacune de ses extrémités à deux tubes en tygon (Sigmamotor) de $2 \mathrm{~cm}$ de long. A 
l'extrémité du tube d'acier qui plonge dans le rumen, une bourse est fixée par une surliure très serrée faite avec un fil de nylon. Cette bourse est constituée d'un parallélépipède $(2,7 \times 6 \times 10 \mathrm{~cm})$ en mousse de polyuréthane à mailles fines (du type

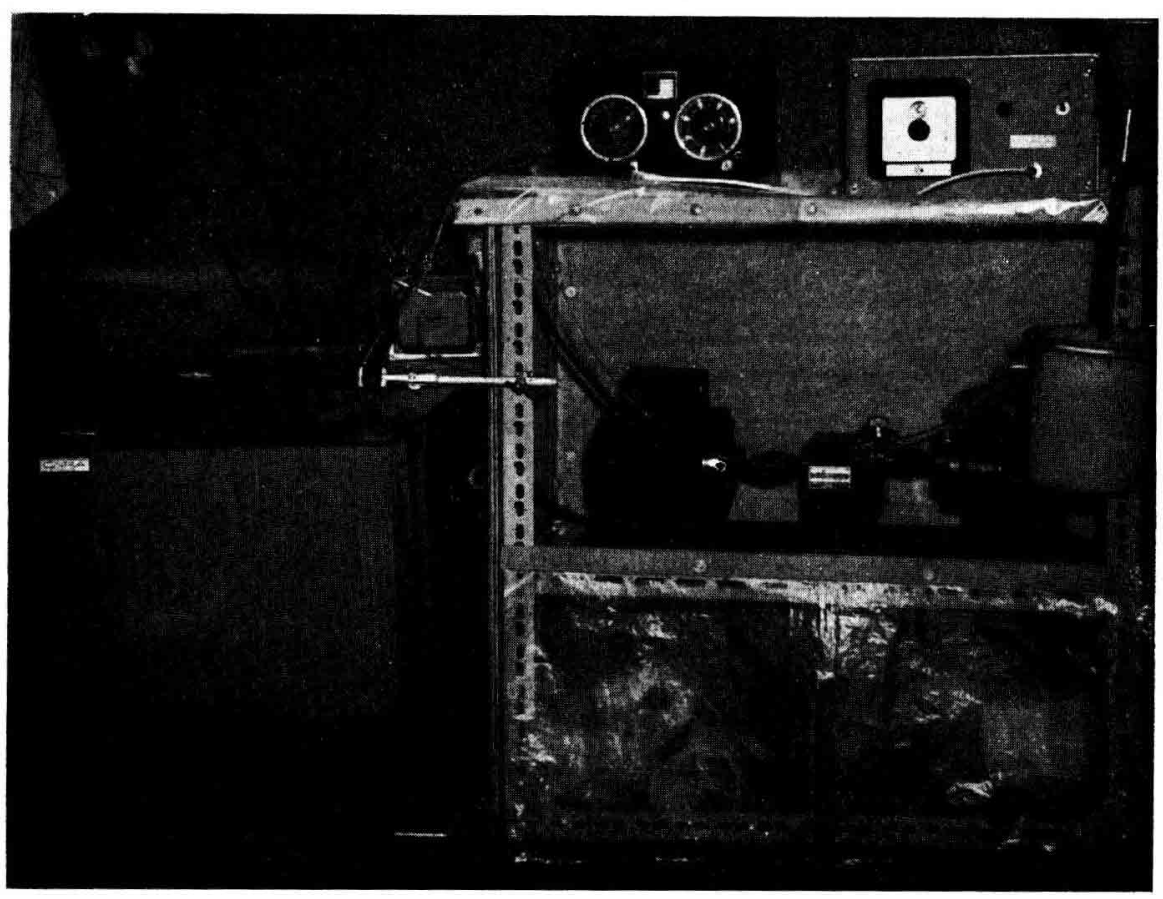

Fig. 2... Vue d'cnsemble de l'appareillage

(it gauche. l'enceinte refroidie contenant le collecteur de frations)

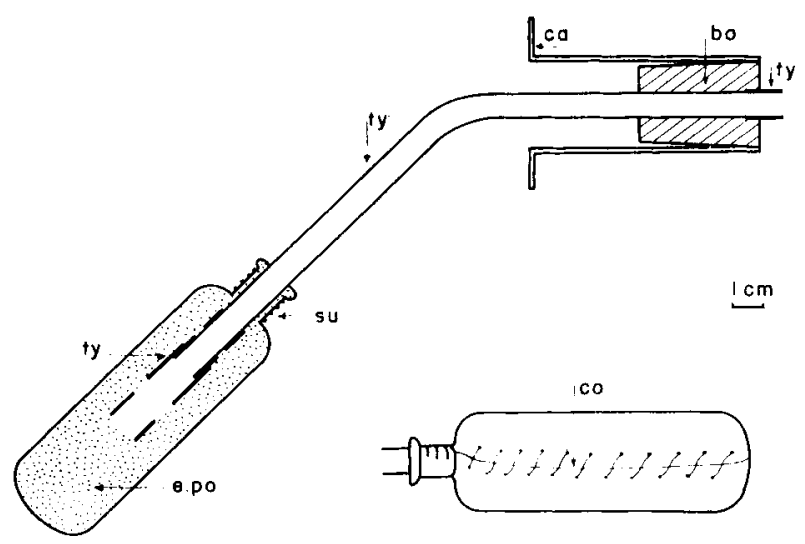

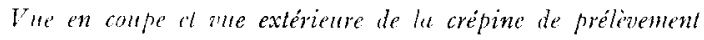

bo bouchon de la canule

ty tube de tygon de la pompe

ca canule

tu tube d'acier inoxydable su surliure de fixation du polyurcthane

c.po ćponge en polyurethane

co couture di l'éponge. 
utilisé pour le rembourrage des sièges) ; les coutures sont faites sans serrer avec du fil de tresse de nylon. La bourse doit rester très souple et très élastique. A son autre extrémité, le tube d'acier traverse un bouchon qui permet de fixer l'ensemble sur la canule et d'en obturer l'orifice avec une étanchéité totale. L'extrémité extérieure du tube de tygon est raccordée au tube de la pompe.

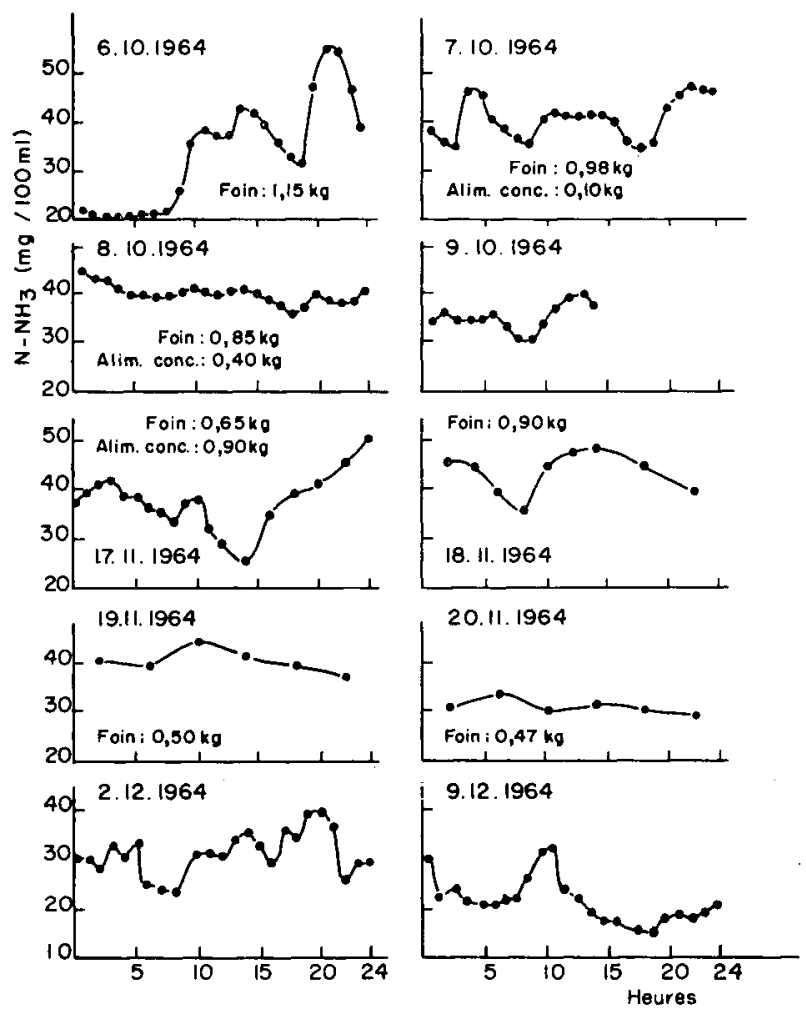

Varialions des leneurs en ammoniac des jus de rumen d'une brebis consommant un foin de luserne et un aliment concentré ad libitum

(trois distributions par jour it 7 h. 30, I I h. 30 et 17 h. 30)

loin :

matières azotées totales 15,3 p. 100 M.S. cellulose brute 26,6 p. 100 Y.S.

liment concentré :

matières azoties totales 19,3 1), 100 M.S.

\section{Discussion}

La crépine peut être laissée plusieurs semaines sur l'animal, sans inconvénient. Compte tenu de la surface d'absorption et du faible débit, il n'y a pas de colmatage de la mousse de polyuréthane avant plusieurs semaines (au moins 3) pour la plupart des régimes. Nous avons eu des obstructions plus rapides ( 8 jours) avec des veaux qui recevaient un aliment broyé et aggloméré contenant $30 \mathrm{p}$. Ioo de farine de luzerne. Il importe que la canule soit bien fixée pour éviter que le tube d'acier ne se déplace, 
entraîné par les mouvements du contenu. Des repaires doivent être effectués pour détecter les déplacements du tube.

Dans la partie du sac ventral où nous effectuons nos prélèvements, nous sommes dans la zone où le liquide est le plus abondant et le plus souvent renouvelé. Entre la pompe et le collecteur de fractions, on peut inclure une cellule à électrodes permettant d'enregistrer le $\mathrm{pH}$ de chaque échantillon prélevé.

Cette méthode permet de prélever nuit et jour pendant de longue périodes le jus de rumen sans tracasser l'animal et sans changer les conditions de la digestion (anaérobiose). Or, si l'on veut effectuer des études cinétiques, il est indispensable de ne pas troubler le processus fermentaire.

I,e graphique donne quelques exemples de courbes obtenues en dosant l'ammoniac dans les échantillons ainsi prélevés. Sur ces courbes un point n'est jamais isolé, ce qui peut faire penser que l'échantillonnage est satisfaisant. L'interprétation des courbes n'est d'ailleurs possible que si l'on connait le comportement alimentaire des animaux :

- la consommation d'eau, de foin et d'aliment concentré ;

- la quantité consommée et la répartition de celle-ci. 